\title{
SUPPORTING INFORMATION \\ Direct Generation of Internally Functionalized Nanoporous Polymers: Design of Polymerizable Porogens
}

\author{
Rounak Jana and S Ramakrishnan*
}

\section{Experimental section}

Materials

Polyethylene glycol monomethyl ether (MPEG) 350, 750 and 2000 are purchased from Alfa aesar and dried under vacuum at $90^{\circ} \mathrm{C}$ before use. N-Bromo succinimide (NBS) is purchased from Aldrich and recrystallized from water before use. AIBN is purchased from Aldrich and recrystallized from hot methanol before use. P-toluic acid, triphenyl phosphine $\left(\mathrm{PPh}_{3}\right)$, formaldehyde (37\% solution in water) is purchased from spectrochem and used without further purification. Divinyl benzene (technical grade 80\%) and styrene (99.9\%) are purchased from Aldrich and distilled to remove the quenchers before use. Solvents were purchased from MERCK Chemicals and dried, where necessary, using standard procedures.

\section{Synthesis of monomers}
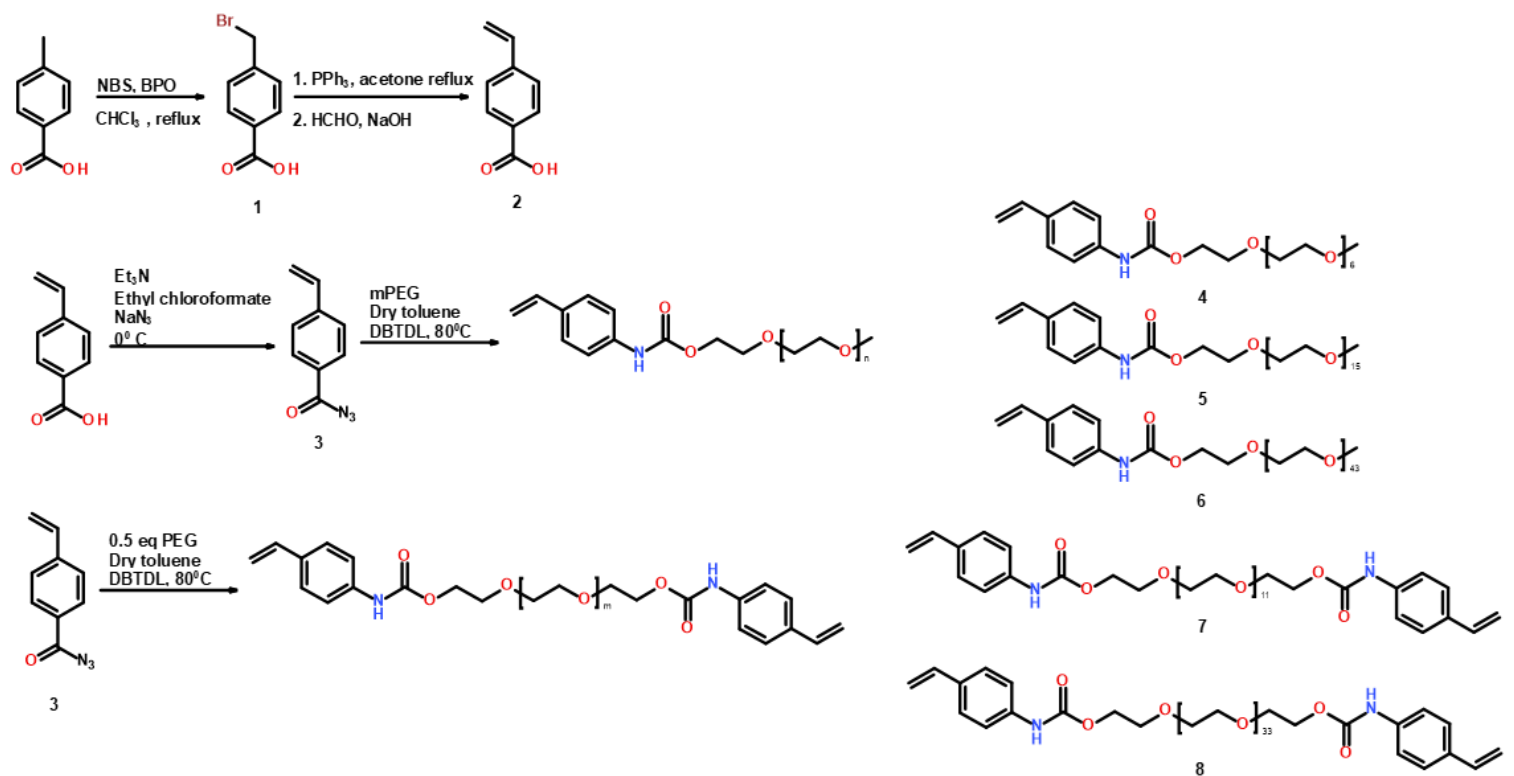

Scheme S1. Synthesis of polymerizable porogens (PolyPo) with varying chain lengths. 


\section{Synthesis of 4-bromomethylbenzoic acid (1)}

$6.68 \mathrm{~g}$ of p-toluic acid (49 mmol), $10.5 \mathrm{~g}$ of N-bromosuccinamide (NBS, $59 \mathrm{mmol}$ ) and 120 $\mathrm{mg}$ of benzoyl peroxide (BPO,0.49 mmol) were mixed together in $85 \mathrm{ml}$ of $\mathrm{CHCl}_{3}$. The reaction mixture was refluxed for $14 \mathrm{hrs}$ under vigorous stirring and then cooled down to r.t. The white precipitate was filtered, washed two times with hot water and then recrystallized from boiling methanol. (yield $=70 \%$ )
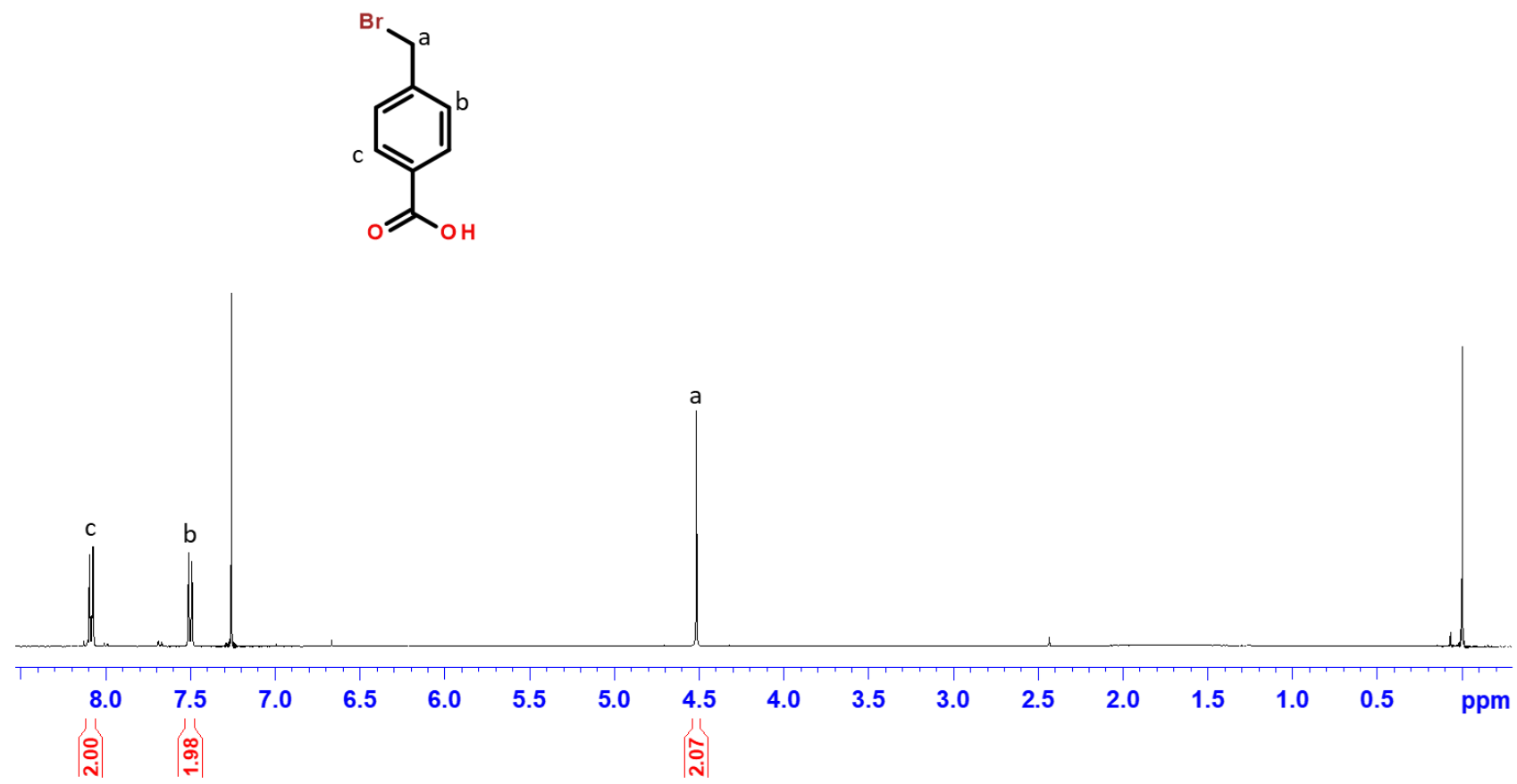

Figure S1. ${ }^{1} \mathrm{H}$ NMR of 4-bromomethylbenzoic acid

\section{Synthesis of 4-vinylbenzoic acid (2)}

$7 \mathrm{~g}$ (32.5 mmol) of 4-bromomethylbenzoic acid and $9.4 \mathrm{~g}$ (35.8 mmol) triphenylphosphine were added to $120 \mathrm{ml}$ of acetone and then refluxed for 6 hours. The mixture was then cooled to room temperature and filtered. The white ppt was then washed with diethyl ether and dried (12.9 g; yield $=90 \%)$. To the phosphonium salt, $85 \mathrm{ml}$ of $37 \%$ formaldehyde solution was added and to this mixture, $9.3 \mathrm{~g}(0.23 \mathrm{~mol})$ of $\mathrm{NaOH}$ in $40 \mathrm{ml}$ of water was added dropwise at r.t; the mixture was stirred for another 2 hours before filtration. The filtrate was then neutralized with $1 \mathrm{~N} \mathrm{HCl}$ till the $\mathrm{pH}$ reaches 1 . The white precipitate formed was then filtered, washed with water and dried under vacuum to yield 3. (Yield 84\%). 

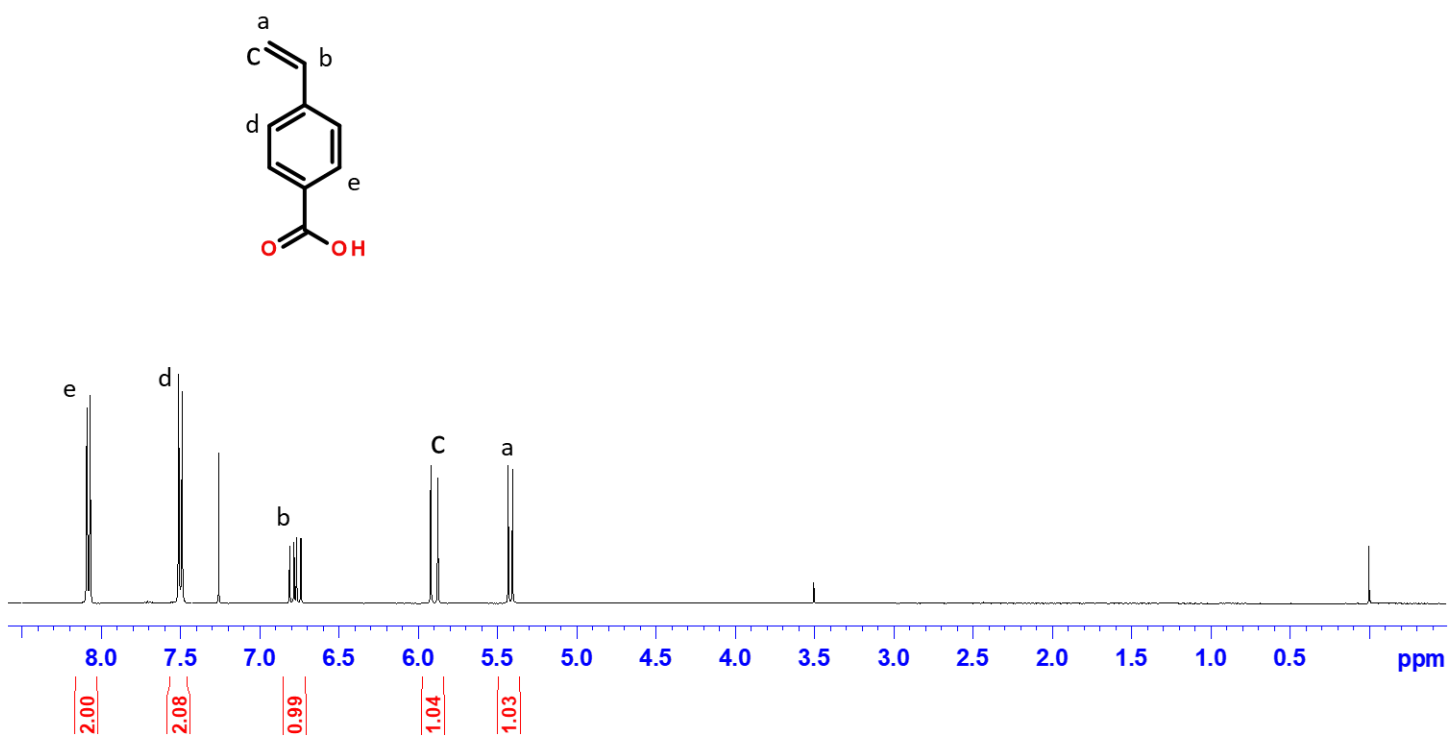

Figure S2. ${ }^{1} \mathrm{H}$ NMR of 4 -vinyl benzoic acid

\section{Synthesis of 4-vinyl benzoyl azide (3)}

$0.7 \mathrm{~g}(4.72 \mathrm{mmol})$ of compound 3 and $0.48 \mathrm{~g}(4.72 \mathrm{mmol})$ triethyl amine $\left(\mathrm{Et}_{3} \mathrm{~N}\right)$ were dissolved in distilled THF. To the solution, $0.72 \mathrm{~g}(6.62 \mathrm{mmols})$ of ethyl chloroformate was added at $0{ }^{\circ} \mathrm{C}$ and stirred for $30 \mathrm{mins}$ at $0{ }^{\circ} \mathrm{C}$. $\mathrm{NaN}_{3}(1.23 \mathrm{~g}, 18.9 \mathrm{mmol})$ in water was added dropwise to the ice-cold reaction mixture and the contents were stirred for 2 hrs at r.t. THF was evaporated keeping the temperature below $40{ }^{\circ} \mathrm{C}$. To the residue, $10 \mathrm{ml}$ of water was added and extracted with $\mathrm{CH}_{2} \mathrm{Cl}_{2}$ several times. The DCM layer was dried using $\mathrm{Na}_{2} \mathrm{SO}_{4}$, evaporated to dryness to yield a white crystalline solid $(0.73 \mathrm{~g}$, Yield $=90 \%)$, whose structure was confirmed by ${ }^{1} \mathrm{H}$ NMR and FTIR. 


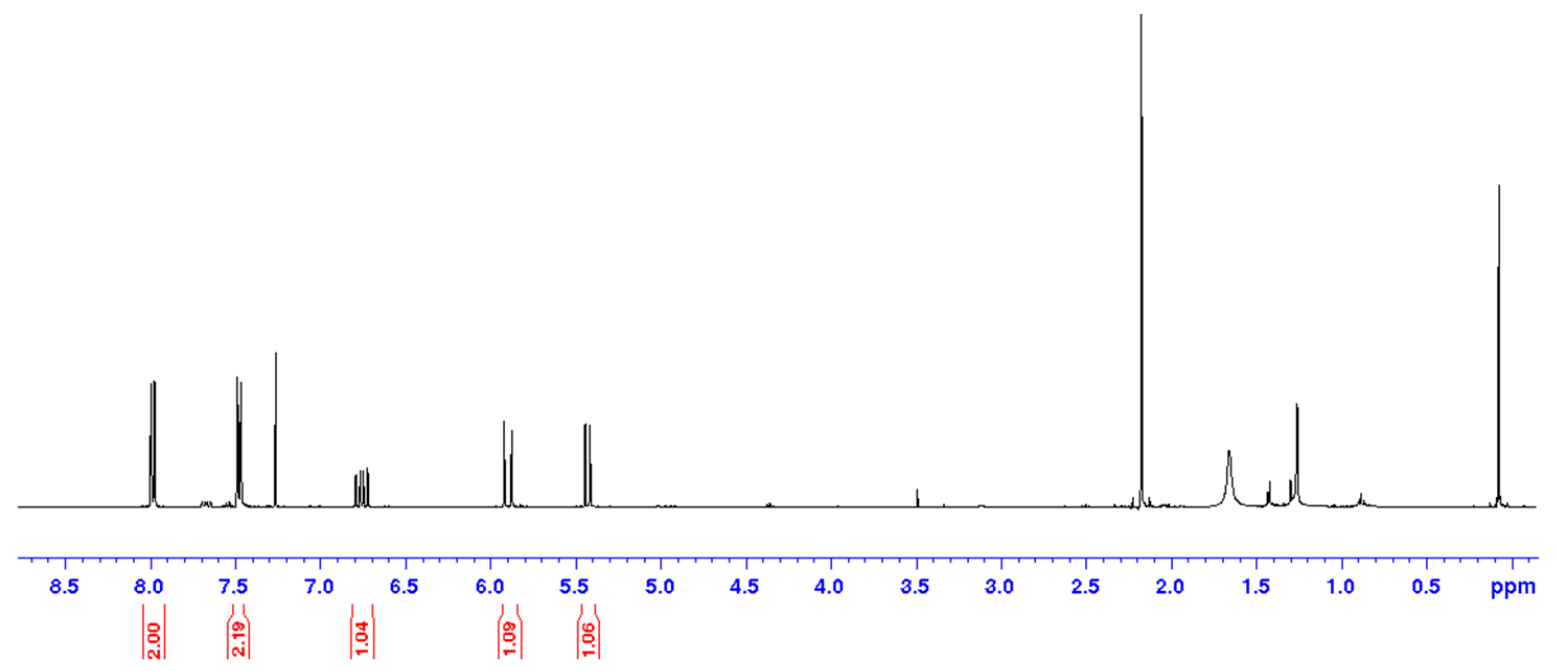

Figure S3. ${ }^{1} \mathrm{H}$ NMR of 4-vinylbenzoyl azide.

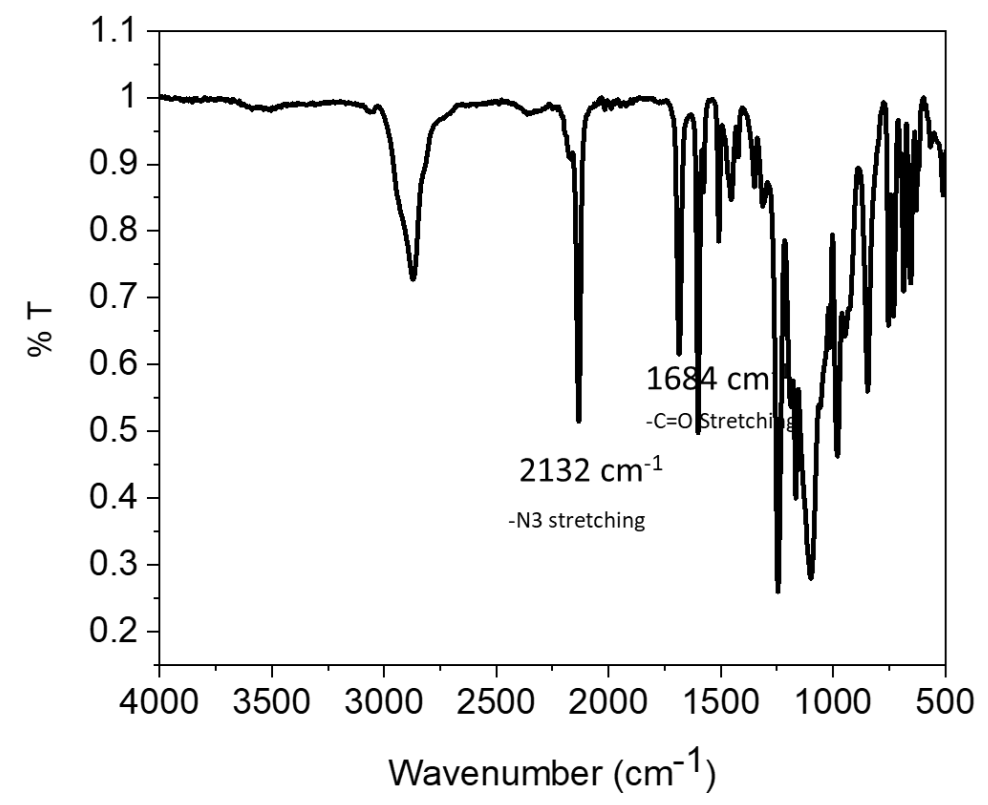

Figure S4. ATR IR of 4-vinyl benzoyl azide. 


\section{Synthesis of St-PEG-350 (4)}

$0.73 \mathrm{~g}(4.21 \mathrm{mmol})$ of 4-vinylbenzoylazide (3) and 1.47g (4.21 mmol) of MPEG 350 were dissolved in dry toluene. To the solution, 2 drops of dibutyl tin dilaurate (DBTDL) was added. The solution was then heated at $80{ }^{\circ} \mathrm{C}$ for 4 days under $\mathrm{N}_{2}$ atmosphere. Then, the reaction mixture was cooled down, toluene was evaporated, and the residue was washed with hexane and dried to get yellowish viscous liquid as product $4(2.0 \mathrm{~g}$, yield $=96 \%)$.

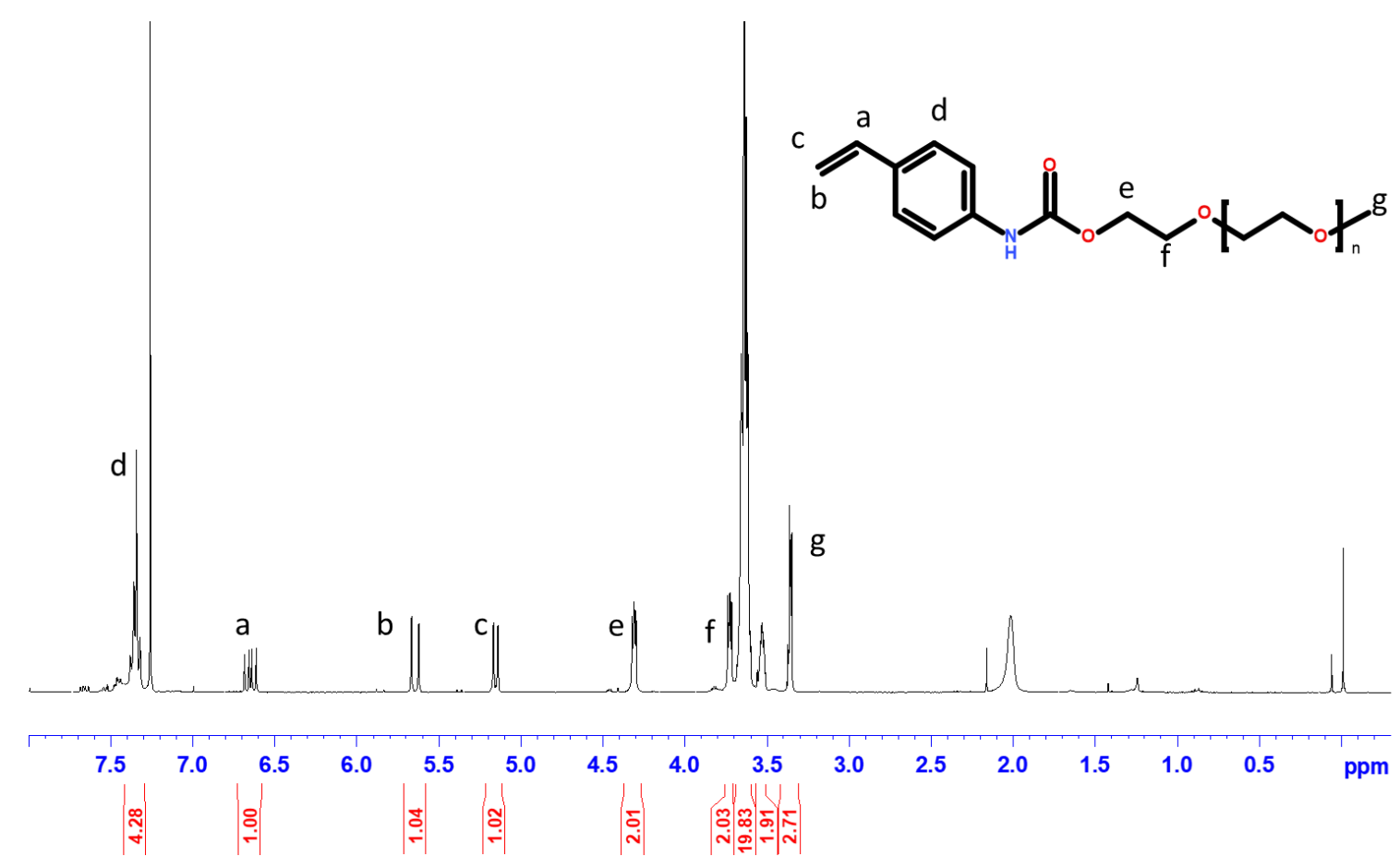

Figure S5. ${ }^{1} \mathrm{H}$ NMR of St-PEG-350; the 1:2 ratio of intensities of peaks $a$ and $e$ helps confirm the structure. 


\section{Synthesis of St-PEG-750 (5)}

$0.7 \mathrm{~g}$ (4.04 mmol) of 4-vinyl benzoyl azide (3) is reacted with 3.03g (4.04 mmol) MPEG 750 following the above-mentioned reaction condition and work up procedure to yield St-PEG$750(3.57 \mathrm{~g}$, yield 97\%).
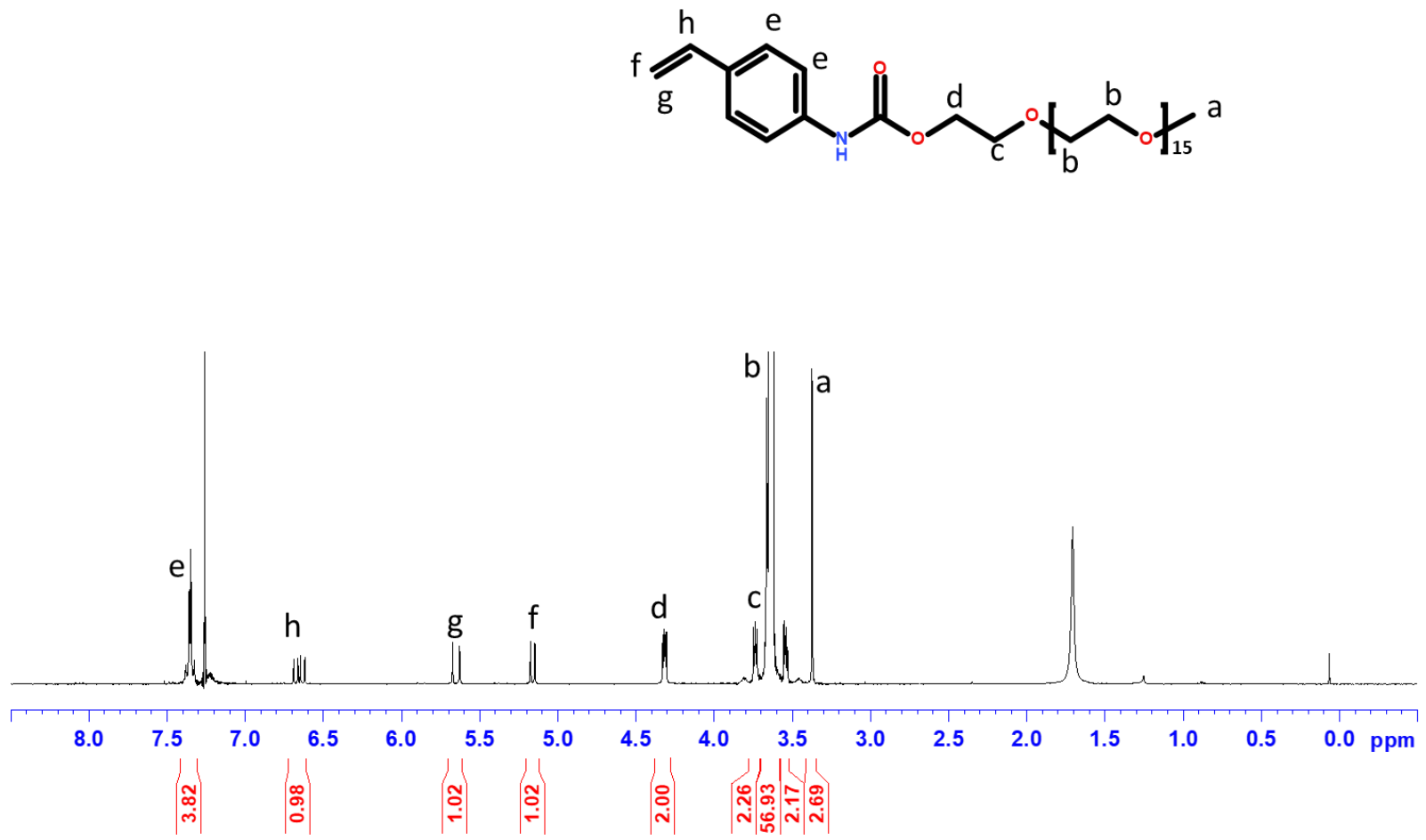

Figure S6. ${ }^{1} \mathrm{H}$ NMR of St-PEG-750; the 1:2 ratio of intensities of peaks $a$ and $e$ helps confirm the structure.

\section{Synthesis of St-PEG-2000 (6)}

$0.16 \mathrm{~g}(0.924 \mathrm{mmol})$ of 4 -vinyl benzoyl azide was reacted with $1.85 \mathrm{~g}(0.925 \mathrm{mmol})$ of MPEG 2000 following the above-mentioned procedure and work up procedure to yield St-PEG-2000 $(1.9 \mathrm{~g}$, yield $=97 \%)$. 


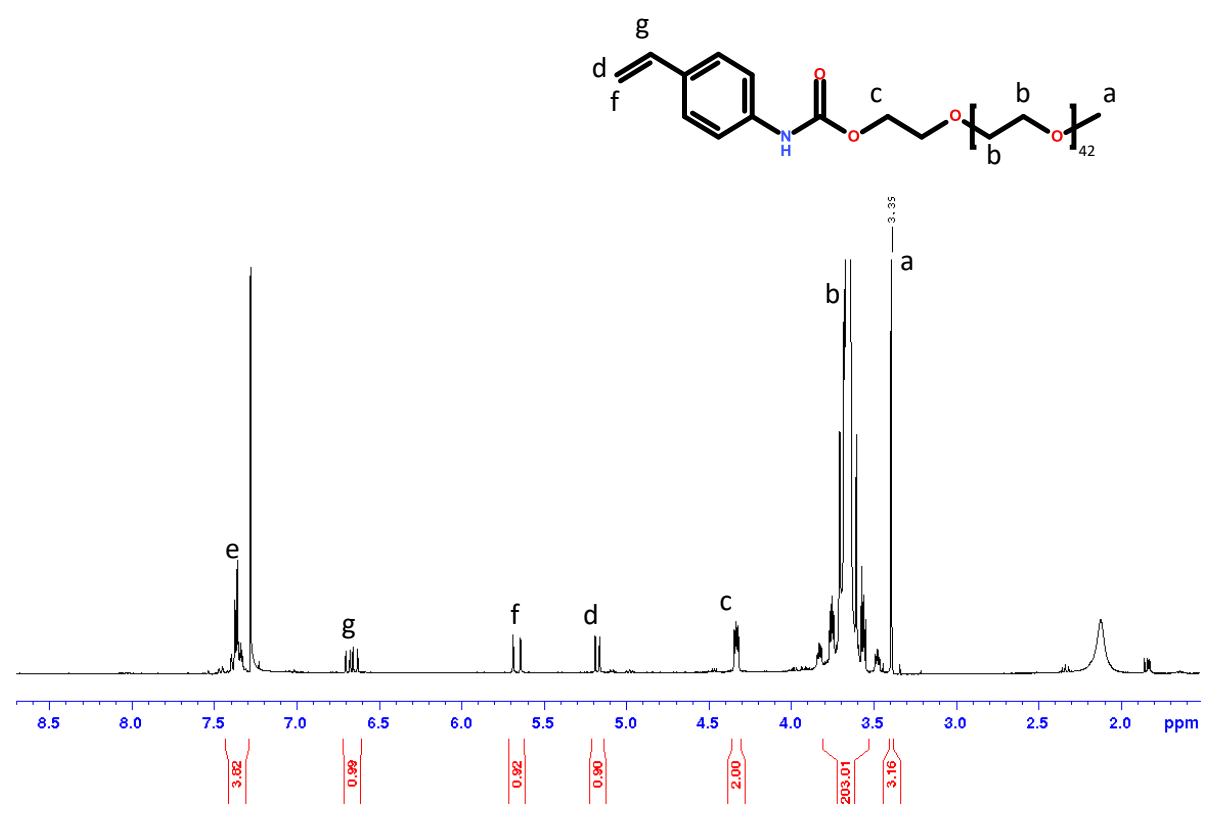

Figure S7. ${ }^{1} \mathrm{H}$ NMR of St-PEG-2000; the 1:2 ratio of intensities of peaks $a$ and $e$ helps confirm the structure.

\section{Synthesis of Di-St-PEG-600 (7)}

$0.51 \mathrm{~g}$ (2.9 mmols) 4 vinyl benzoyl azide (3), 0.8g (1.4 mmols) PEG 600 were dissolved in dry toluene. 2 drops of di butyl tin dilaurate (DBTDL) were added to the reaction mixture and then heated at $80^{\circ} \mathrm{C}$ for 4 days under $\mathrm{N}_{2}$ atmosphere. After that, reaction mixture was cooled down to r.t. and the toluene was evaporated under vacuum. The product was obtained as a yellow viscous liquid after washing in hexane followed by drying under vacuum (1.2g, 92\% yield).

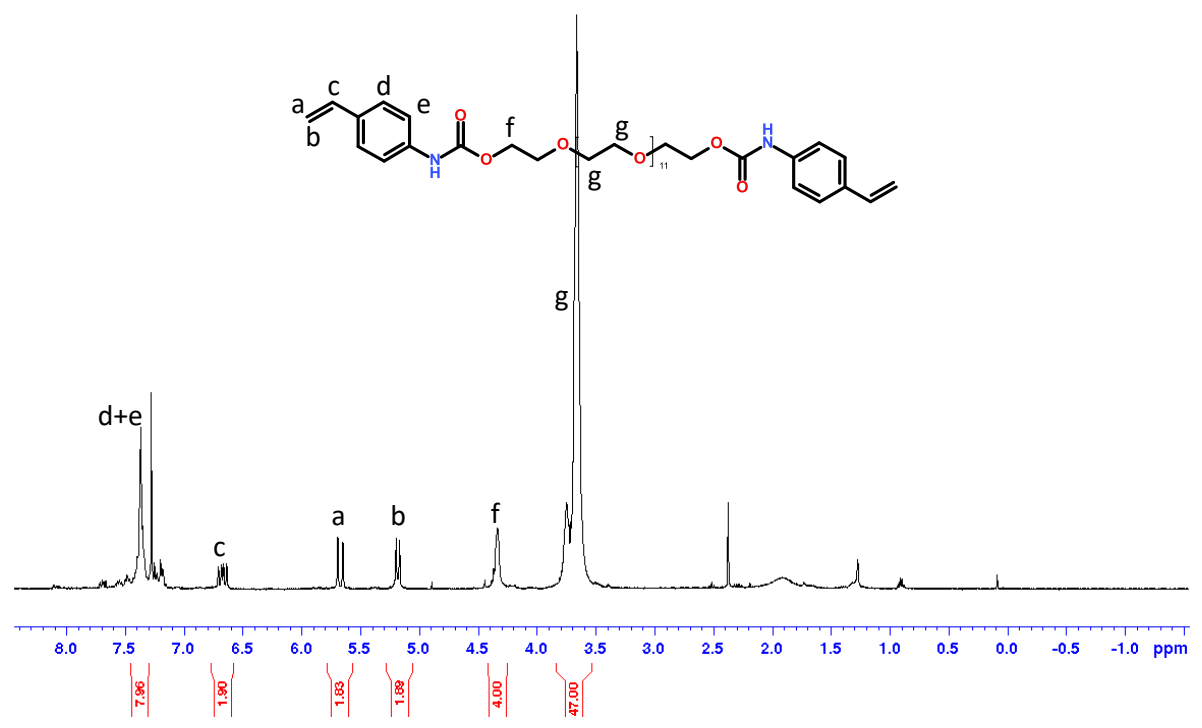

Figure S8. ${ }^{1} \mathrm{H}$ NMR of Di-St-PEG-600; the 1:2 ratio of intensities of peaks $a$ and $f$ helps confirm the structure. 


\section{Synthesis of Di-St-PEG-1500 (8)}

$0.88 \mathrm{~g}$ (5.0 mmols) of 4 vinyl benzyl azide (3) was reacted with $3.4 \mathrm{~g}$ (2.3 mmols) of PEG 1500 following the above-mentioned procedure and work up procedure to yield 8 as a yellow solid (3.7g, yield 90\%).

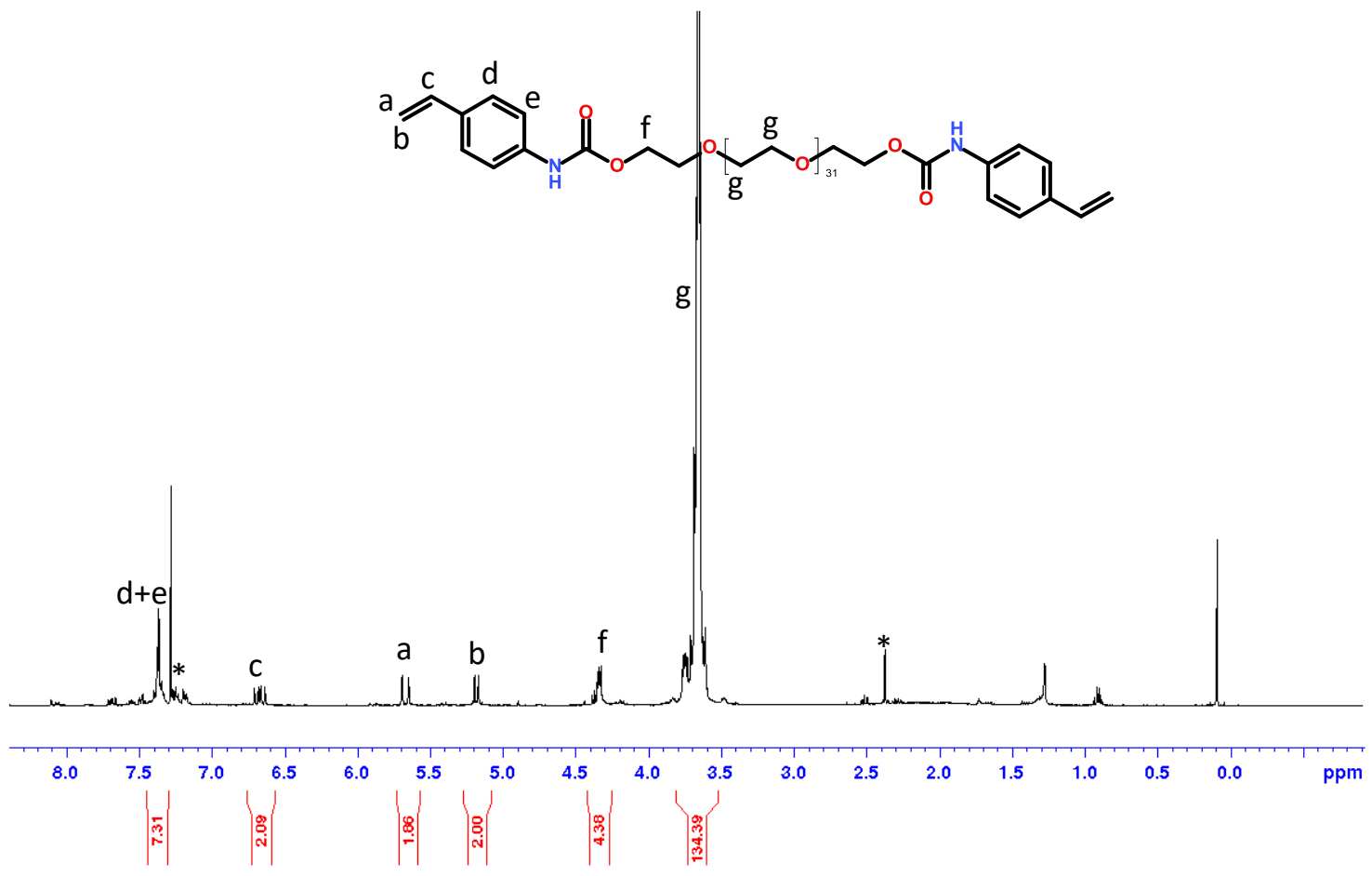

Figure S9. ${ }^{1} \mathrm{H}$ NMR of Di-St-PEG-1500

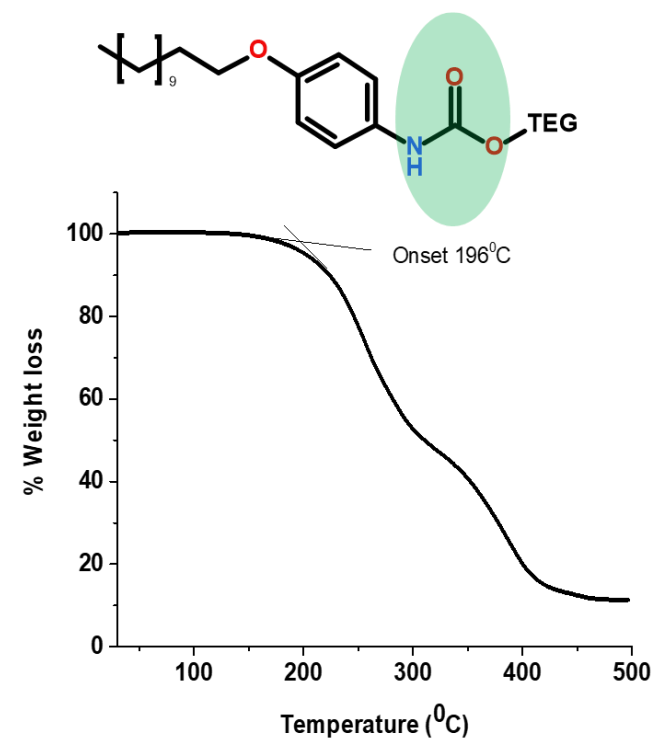

Figure S10. TGA analysis of model compound, the structure of which is shown above. The onset of wt-loss is found to be $196{ }^{\circ} \mathrm{C}$; this is more a reflection of the volatilization of the cleaved moiety rather than the temperature of cleavage of the urethane linkage, which is lower as per IR studies. 


\section{Synthesis of St-co-St-PEG-350}

$0.20 \mathrm{~g}(1.92 \mathrm{mmol})$ styrene and $0.10 \mathrm{~g}(0.213 \mathrm{mmol})$ of St-PEG-350 were dissolved in $4 \mathrm{ml}$ of $\mathrm{CHCl}_{3}$ along with $3 \mathrm{mg}$ of AIBN. The reaction mixture was purged with $\mathrm{N}_{2}$ for 5 mins to remove the dissolved oxygen. Then the reaction vessel was capped under $\mathrm{N}_{2}$, heated at $65{ }^{\circ} \mathrm{C}$ for 6 hours.

After cooling, the reaction mixture was concentrated, reprecipitated from methanol 3 times, and dried to obtain a yellowish polymer.

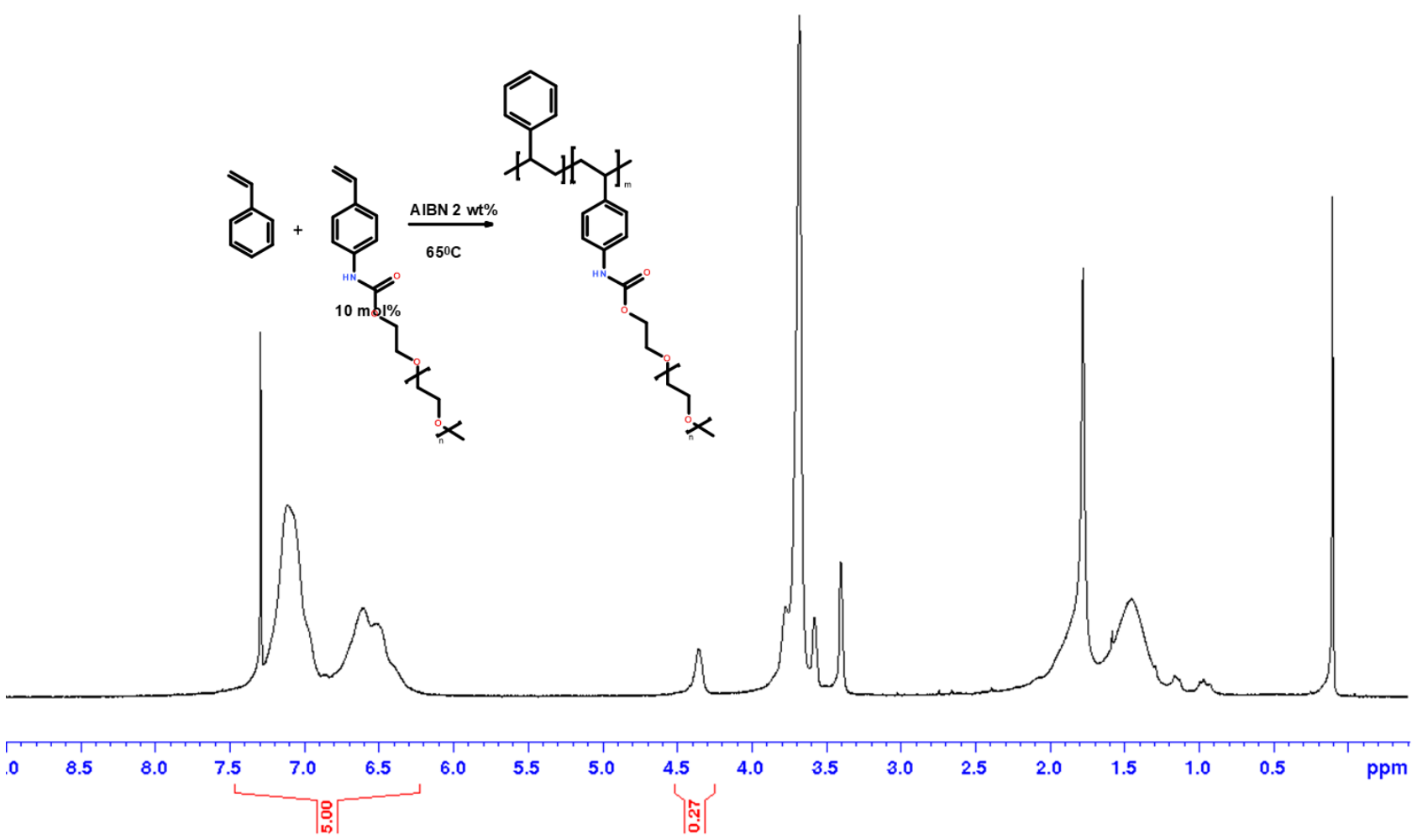

Figure S11. ${ }^{1} \mathrm{H}$ NMR of the St-co-St-PEG-350 copolymer. The copolymer composition, estimated from the relative intensities of entire aromatic region (i.e., 5) and the methylene protons at $\sim 4.3 \mathrm{ppm}$ (i.e., 0.27); the amount of St-PEG-350 incorporated was found to be 12.3 mole-percent, as against 10 mole-percent in the feed. 


\section{General procedure for the preparation of the crosslinked monoliths}

In each case, the total amount of reaction mixture was kept constant at $0.60 \mathrm{~g}$, but the relative amount of DVB to St-PEG- $X(X=350,750,2000)$ was varied according to table 1 . In a typical polymerization, DVB and St-PEG- $X$ was mixed together to form a homogeneous clear solution in a vial. To the reaction mixture $10 \mathrm{mg}$ of benzoyl peroxide (BPO) and $40 \mathrm{mg}$ of TEMPO was added and stirred for 10 mins (for entry 1, no TEMPO was used). The reaction mixture was then transferred to a polymerization tube, where it was degassed by three freeze-pump-thaw cycles. Following degassing, the reaction vessel was vacuum sealed and kept in a tubular furnace at $130{ }^{\circ} \mathrm{C}$ for 72 hours until a clear crosslinked monolith is formed. The monolith was retrieved after breaking the glass tube and was then washed with chloroform for $12 \mathrm{~h}$ prior to extraction to get rid of any unreacted monomers due to vitrification.

\section{General extraction procedure}

The crosslinked monolith was ground to a powder first and taken in a round bottom flask, into which $20 \mathrm{ml}$ of DMSO, $2 \mathrm{ml}$ of $1 \mathrm{~N} \mathrm{NaOH}$ was added; the reaction mixture was degassed by three freeze-pump-thaw process and heated to $155^{\circ} \mathrm{C}$ under $\mathrm{N}_{2}$ atmosphere for 3 days. The powder suspension was isolated by centrifugation, washed thrice with a water-methanol mixture, followed by treatment in boiling acetone for 12 hours; this was to ensure complete removal of the porogen. Thereafter, the powder was isolated by centrifugation and dried in the vacuum oven at $80{ }^{\circ} \mathrm{C}$ for 12 hours. FTIR and ${ }^{13} \mathrm{C}$ MAS NMR spectra of the powder were recorded and are shown in Figures S10 and S11.

To identify the composition of the extracted solution, the supernatant was concentrated at 90 ${ }^{\circ} \mathrm{C}$ under high vacuum, neutralized with $1 \mathrm{~N} \mathrm{HCl}$ and then extracted with dichloromethane. The DCM layer was collected, dried with $\mathrm{Na}_{2} \mathrm{SO}_{4}$ and concentrated; the NMR spectrum of the residue was recorded to identify its composition, and is shown in Figure S10. 
a

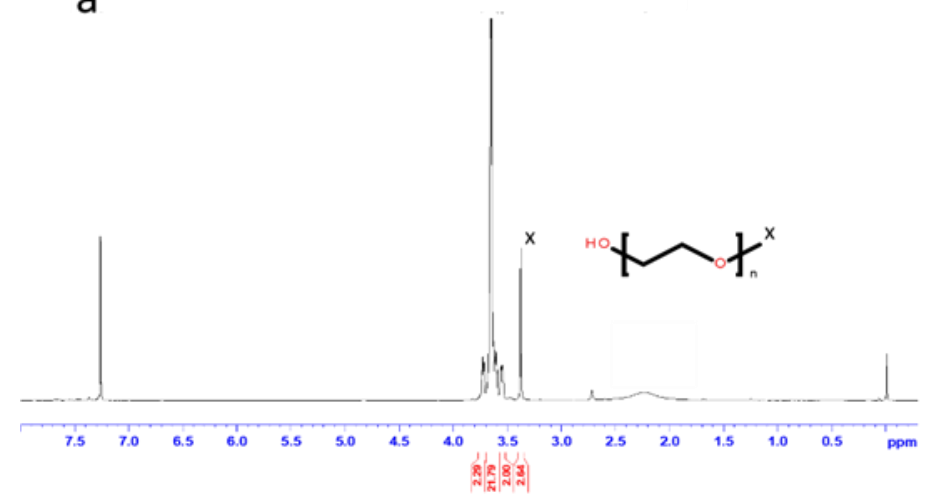

$\mathrm{b}$

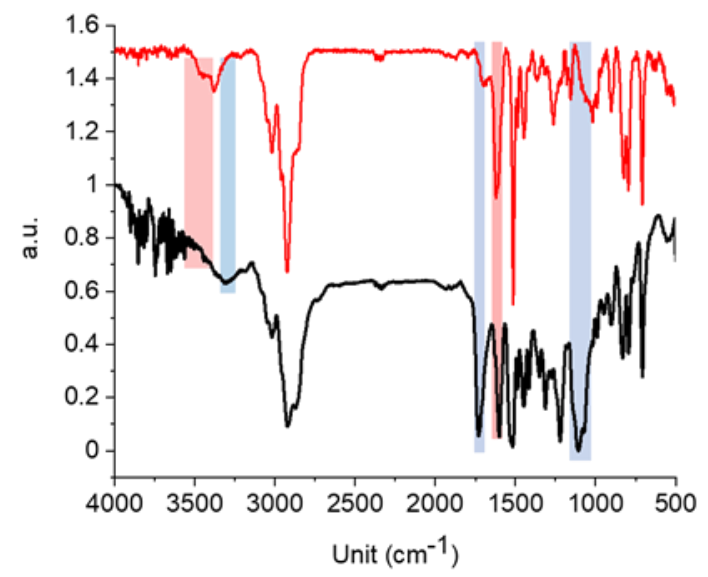

Figure S12. (a) ${ }^{1} \mathrm{H}$ NMR of the DMSO water extract; (b) FTIR spectrum of the crosslinked matrix of 60St350 before (black) and after DMSO-water treatment (red).

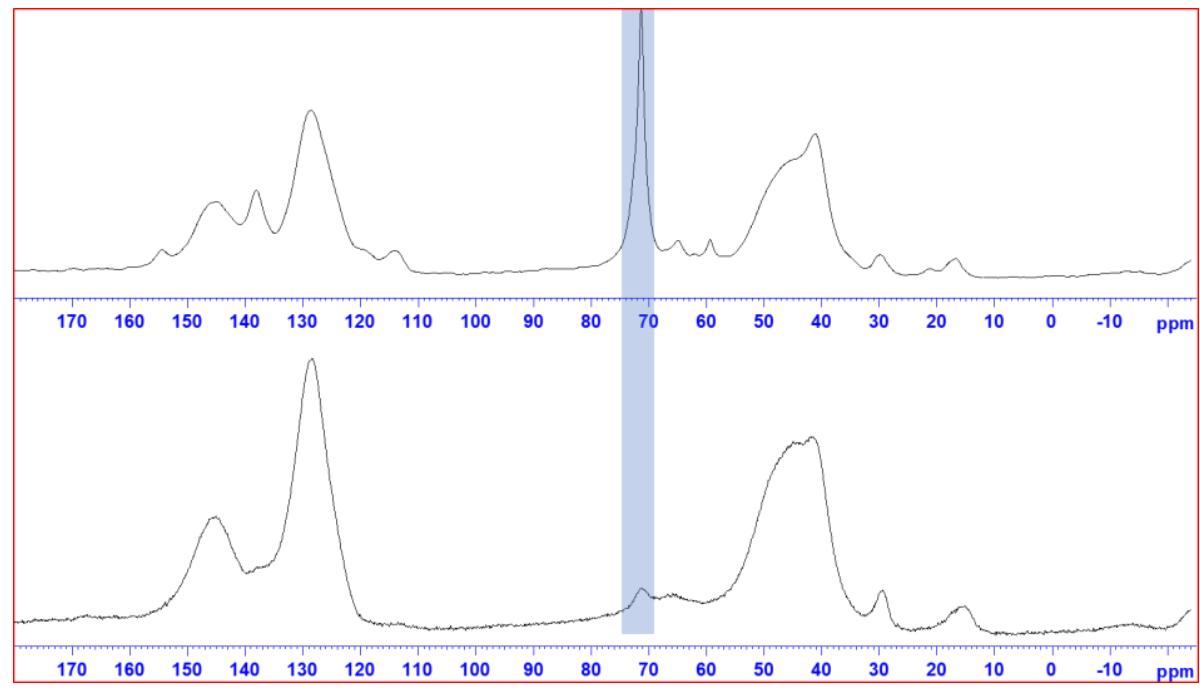

Figure S13. ${ }^{13} \mathrm{C}$ MAS spectra of the crosslinked matrix before (top) and after (bottom) solvothermal of 50St350. The peak around $70 \mathrm{ppm}$ that is due to the carbon in the MPEG is seen to disappear nearly completely. In addition, changes in the low-field region reflects the transformation of the urethane to an amine; specifically, the peak at $\sim 155 \mathrm{ppm}$ due to the urethane carbonyl has completely disappeared. 


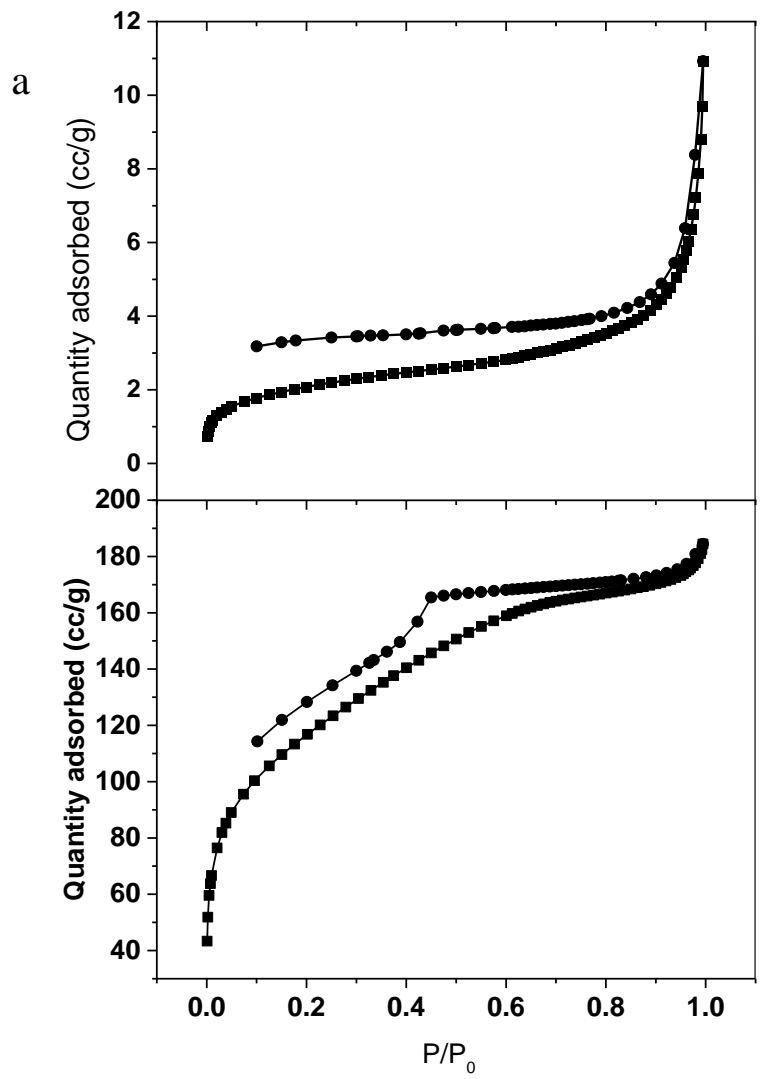

$\mathrm{b}$
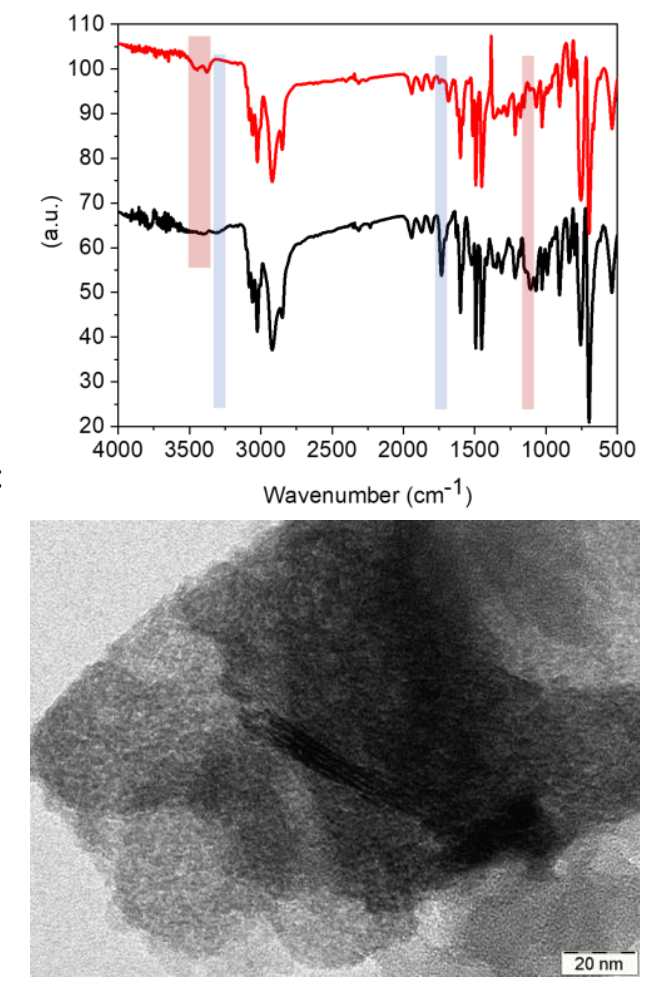

Figure S14. (a) Comparison of BET isotherms of 50St350FRP (i) and 50St350NMP (ii); (b) F-IR spectra before and after extraction; (c) TEM image of a representative sample 50St750NMP (Scale bar $20 \mathrm{~nm}$ ); since the pore size is very small the image does not clearly reveal the morphology.
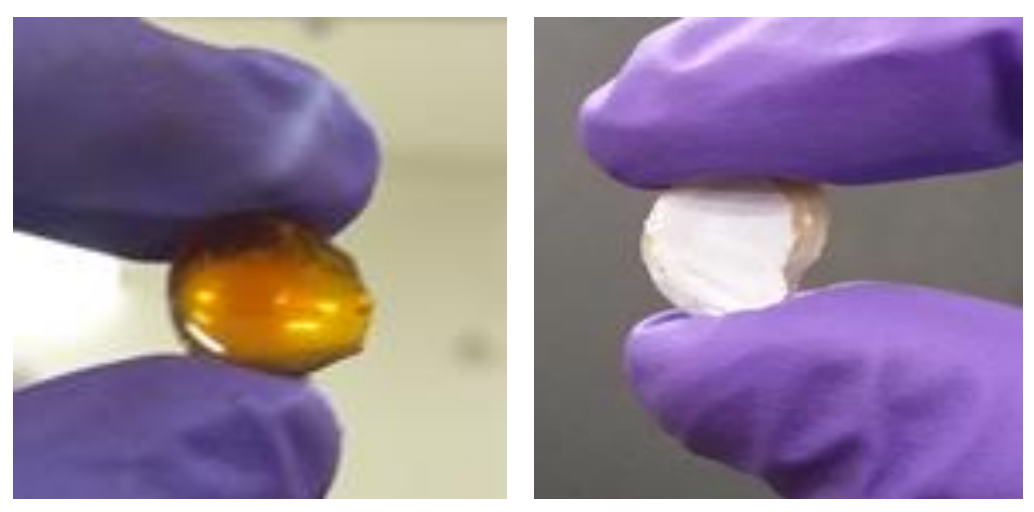

Figure S15. Photographs of 50St350 (left) and 50PEG350 (right) samples, as prepared; the latter was prepared using free MPEG350 and reveals macrophase separation, as evident from the opacity. 


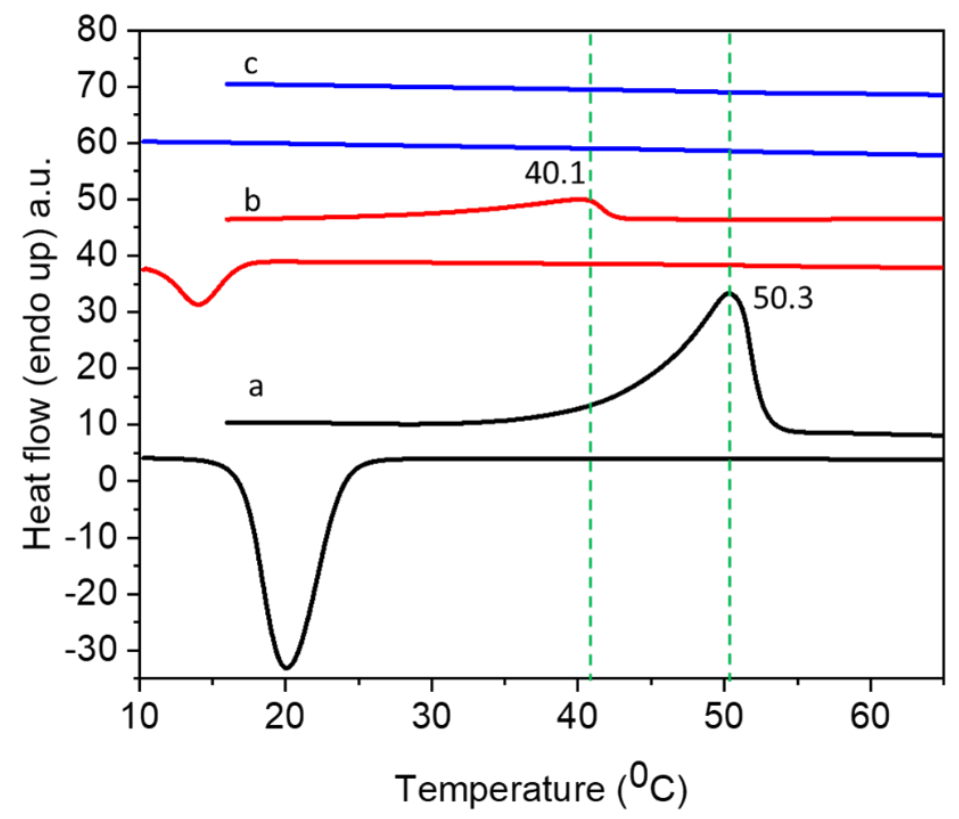

Figure S16. DSC thermogram of a) St-PEG-2000 (in black), b)50St2000 before etching (in red) and c) 50 St2000 after etching (in blue). Before etching the melting peak of the crosslinked matrix around $40.1^{\circ} \mathrm{C}$ indicate the $\mathrm{T}_{\mathrm{m}}$ of microphase separated PEG domain which is absent after extraction. Reduction of $\mathrm{T}_{\mathrm{m}}$ in the crosslinked matrix compared to the pure monomer $\left(\mathrm{T}_{\mathrm{m}}\right.$ $=50.3^{0} \mathrm{C}$ ) is due to the restricted motion of the PEG in the crosslinked matrix.
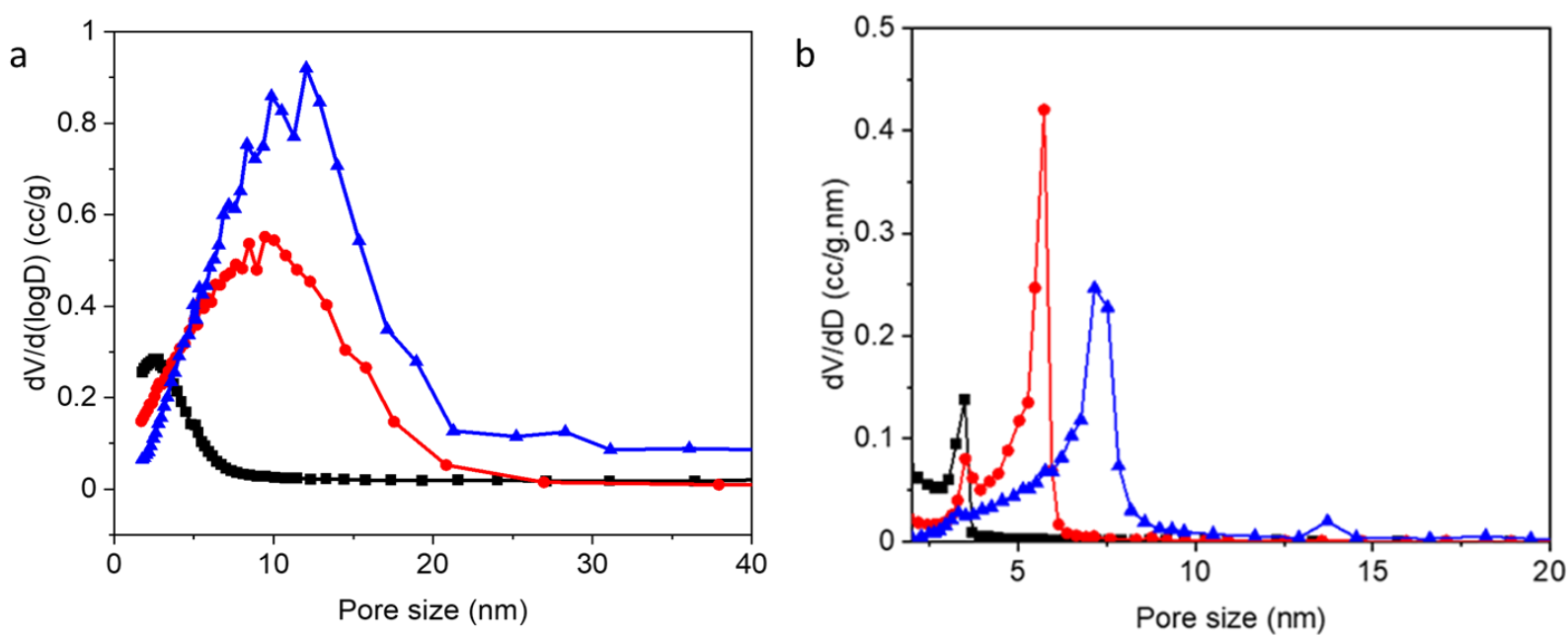

Figure S17. Pore size distribution as a function of MPEG segment length in the PolyPo. BJH pore size distribution calculated from a) adsorption isotherm, b) desorption isotherm for 50St350 (in black), 50St750 (in red) and 50St2000 (in blue). A change in average pore size with MPEG segment length is evident. The average pore size calculated from adsorption isotherm by this method is reported in table 1 and has been used to plot figure $5 \mathrm{c}$. 


\section{Estimation of amine groups in the porous solids by conductometric titration}

Conductometric titrations were carried out with the amine functionalized samples to estimate the accessible amine group. For this, $0.01 \mathrm{~N} \mathrm{NaOH}$ and $0.064 \mathrm{~N} \mathrm{HCl}$ solutions were prepared in 1:1 $\mathrm{MeOH} /$ Water mixture. First, $0.064 \mathrm{~N} \mathrm{HCl}$ solution was prepared and standardize with standard $0.064 \mathrm{~N} \mathrm{Na}_{2} \mathrm{CO}_{3}$; likewise, the $0.018 \mathrm{~N} \mathrm{NaOH}$ was standardized against $0.064 \mathrm{~N} \mathrm{HCl}$. For the preparation of all the solutions milli-q water was used (conductance $=0.055 \mu \mathrm{S}$ ). In each experiment, $50 \mathrm{mg}$ of the sample was dispersed with $5 \mathrm{ml}$ of $0.064 \mathrm{~N} \mathrm{HCl}$ and stirred overnight; $5 \mathrm{~mL}$ of blank solution containing no sample was also set aside. After overnight stirring, the excess $\mathrm{HCl}$ was then back titrated with $0.018 \mathrm{~N} \mathrm{NaOH}$, while the conductivity of the solutions was monitored with the a conductometer; to permit complete equilibration, especially nearing the equivalence point, 10 mins of stirring was done after addition of each portion, prior to measurement of the conductivity. The conductometric titration plots are shown in figure S16. It is seen that the conductivity first drops because of the consumption of excess $\mathrm{HCl}$, after which there is a flat portion, which reflects the neutralization of the immobile $\mathrm{HCl}$ bound to the amines in the porous solid; this is followed by a rise, which is due to the increase in the $\mathrm{NaOH}$ concentration. The amount of $\mathrm{NaOH}$ consumed in the flat region of the plot is taken to represent the moles of amine group present in the porous polymer. As evident from the plot, the blank sample which does not contain the porous polymer, exhibits a standard plot without a flat region; and the neutralization point matches well with the point at which the rise in conductivity happens in the other samples. 

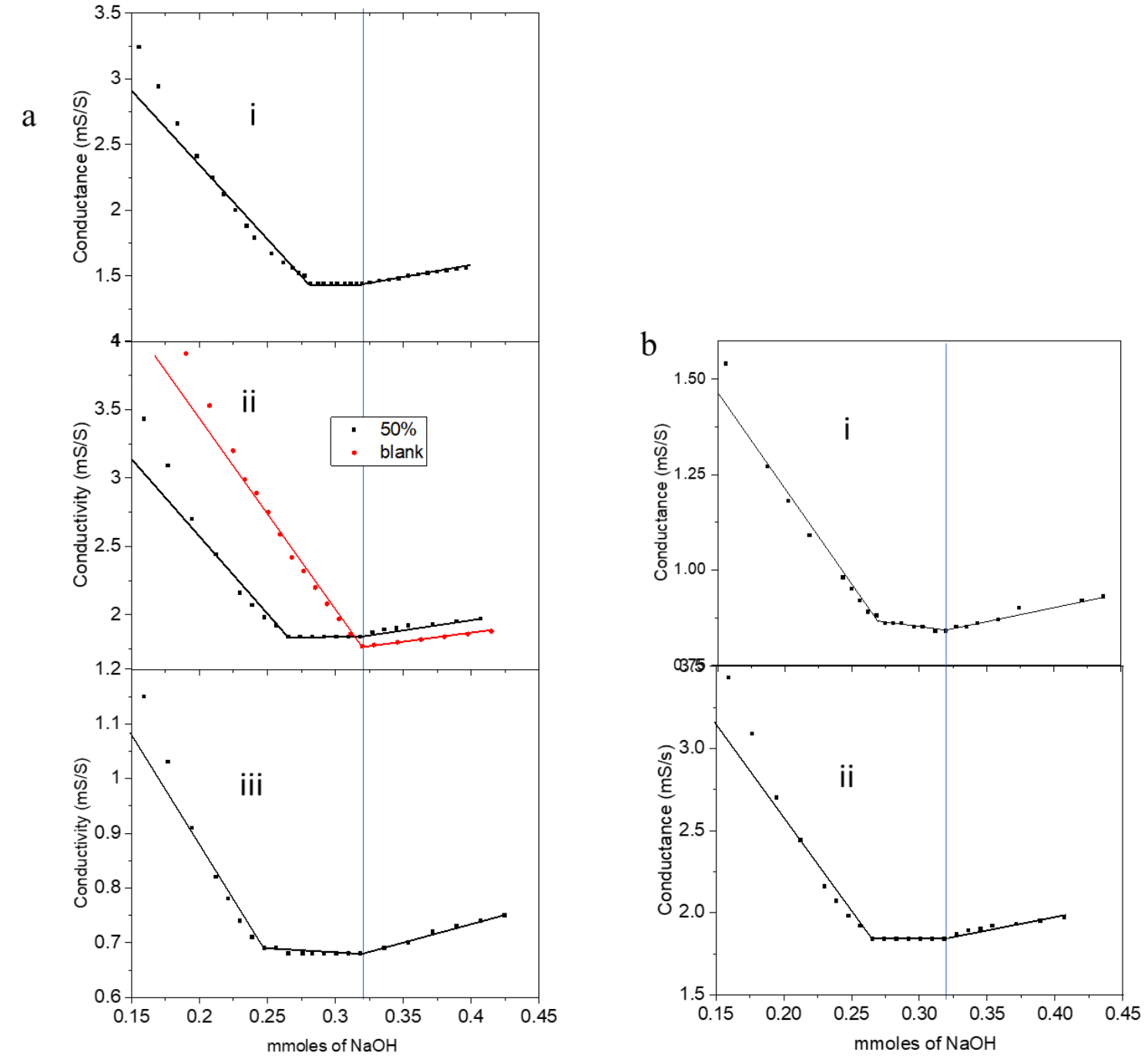

Figure S18. Conductometric titration in 50:50 (v/v) MeOH-water with (a) (i) 40St350 (ii) $50 \mathrm{St} 350$ (iii) $60 \mathrm{St} 350$ and with blank (in red) The steep decrease in conductivity the initial stage reflects the loss of excess $\mathrm{H}^{+}$ions; the flat region corresponds to the neutralization of immobile protons associated with the amines $\left(\right.$ as $\left.-\mathrm{NH}_{3}{ }^{+}\right)$in the crosslinked polymer; and the slow rise thereafter reflects the increase in $\mathrm{OH}^{-}$ions. It is important to note the near perfect coincidence of the second increase with that of a blank titration (in red), which helps reconfirm the validity of this measurement. (b) (i) $50 \mathrm{DiSt} 600$ (ii) $50 \mathrm{St} 350$. The flat region is measured to be comparable suggesting similar number of $-\mathrm{NH}_{2}$ functionality present in two samples. 


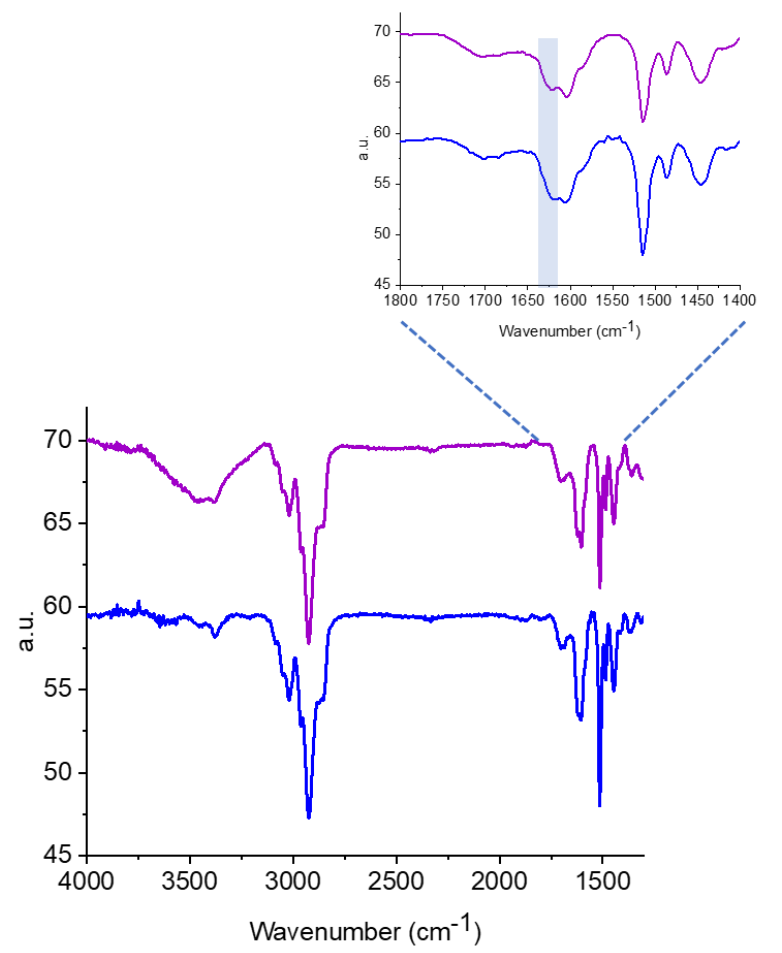

Figure S19. FTIR of 50St350 (in blue) and 50DiSt600 (in violet) normalized w.r.t. -CH stretching. In the inset $1400 \mathrm{~cm}^{-1}$ to $1800 \mathrm{~cm}^{-1}$ is zoomed. The highlighted peak in the inset corresponds to the $-\mathrm{NH}$ bending peak. The integration of the $-\mathrm{NH}$ bending peaks for two samples were found to be almost identical suggesting they contain similar amount of $-\mathrm{NH}_{2}$ groups.

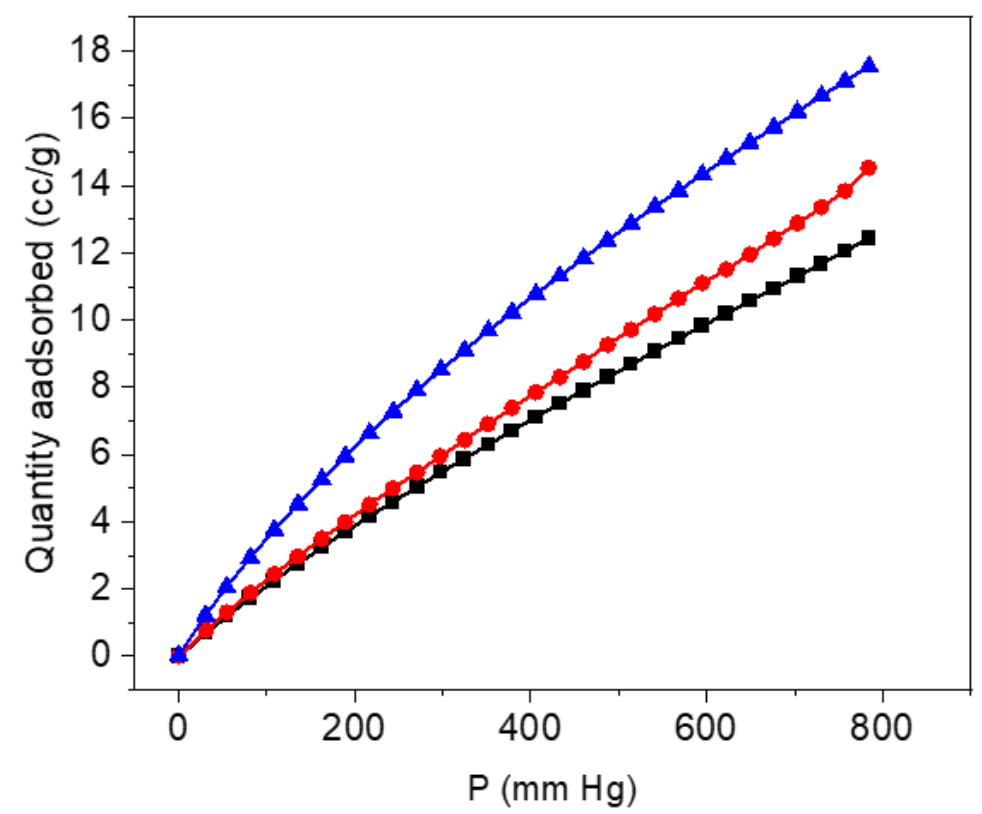

Figure S20. $\mathrm{CO}_{2}$ adsorption @ 298K of 40St350 (in black), 50St350 (in red) and 60St350 (in blue). As the amine content increases amount of $\mathrm{CO}_{2}$ uptake also increases. 

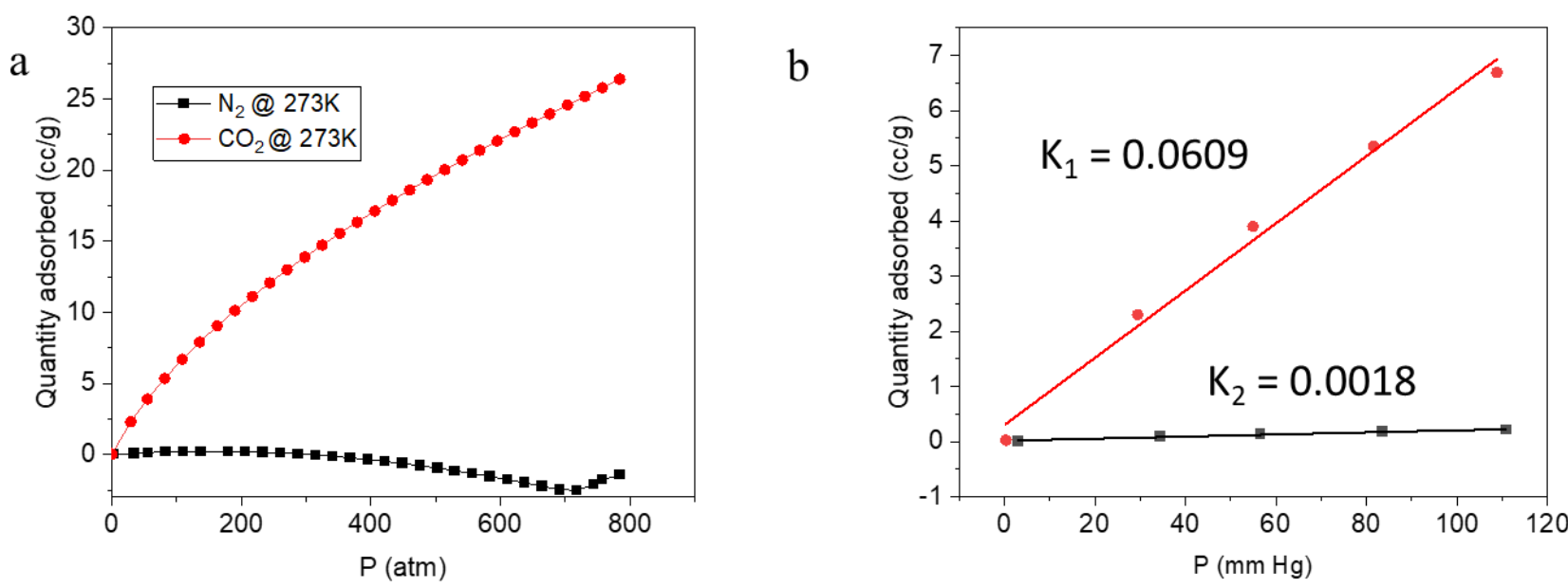

Figure S21. Single gas adsorption isotherm for $60 \mathrm{St} 350$ @ 273K N 2 (in black), $\mathrm{CO}_{2}$ (in red). 


\section{Theoretical estimation of pore volume}

In all calculations, the density of PEG is taken to be $1.125 \mathrm{~g} / \mathrm{cc}$

$\underline{\mathbf{4 0 S t 3 5 0}}$ is synthesized by polymerization of $0.24 \mathrm{~g}$ (40 wt $\%$ ) St-PEG-350 and 0.36g (60 wt $\%$ ) of DVB.

Molecular weight of St-PEG-350 = 495

Weight fraction of PEG in St-PEG-350 $=(350 / 495)=0.7071$

$0.24 \mathrm{~g}$ of St-PEG-350 contains $(0.24 * 0.7071)=0.17 \mathrm{~g}$ of PEG

Assuming full extraction, the volume of PEG present in the sample should be equal to the pore volume.

Volume of PEG present in the sample $=(0.17 / 1.125)=\mathbf{0 . 1 5} \mathbf{c c}$

After the extraction, the remaining weight of the sample $=\{0.36+(0.24-0.17)\}=0.43 \mathrm{~g}$

Theoretical pore volume of 40St350 after extraction $=(0.15 / 0.43)=\underline{\mathbf{0 . 3 4 8}} \mathbf{c c} / \mathbf{g}$.

$\underline{\mathbf{5 0 S t 3 5 0}}$ is synthesized by polymerization of $0.3 \mathrm{~g}(50 \mathrm{wt} \%)$ St-PEG-350 and $0.3 \mathrm{~g}(50 \mathrm{wt} \%)$ of DVB.

Molecular weight of St-PEG-350 = 495

Weight fraction of PEG in St-PEG-350 $=(350 / 495)=0.7071$

$0.3 \mathrm{~g}$ of St-PEG-350 contains $(0.3 * 0.7071)=0.21 \mathrm{~g}$ of PEG

Assuming full extraction, the volume of PEG present in the sample should be equal to the pore volume.

Volume of PEG present in the sample $=(0.21 / 1.125)=\mathbf{0 . 1 8 9} \mathbf{c c}$

After the extraction, the remaining weight of the sample $=\{0.30+(0.3-0.21)\}=0.39 \mathrm{~g}$

Theoretical pore volume of $50 \mathrm{St} 350$ after extraction $=(0.189 / 0.39)=\underline{\mathbf{0 . 4 8 4}} \mathbf{c c} / \mathbf{g}$.

$\underline{\mathbf{6 0 S t 3 5 0}}$ is synthesized by polymerization of $0.36 \mathrm{~g}(60 \mathrm{wt} \%)$ St-PEG-350 and $0.24 \mathrm{~g}$ (40 wt $\%)$ of DVB.

Molecular weight of St-PEG-350 $=495$

Weight fraction of PEG in St-PEG-350 $=(350 / 495)=0.7071$

$0.36 \mathrm{~g}$ of St-PEG-350 contains $(0.36 * 0.7071)=0.254 \mathrm{~g}$ of PEG

Assuming full extraction, the volume of PEG present in the sample should be equal to the pore volume.

Volume of PEG present in the sample $=(0.254 / 1.125)=0.226 \mathrm{cc}$ 
After the extraction, the remaining weight of the sample $=\{0.24+(0.36-0.254)\}=0.346 \mathrm{~g}$

Theoretical pore volume of $60 \mathrm{St} 350$ after extraction $=(0.226 / 0.346)=\underline{\mathbf{0 . 6 5 3}} \mathbf{~ c c / g}$.

$\underline{\mathbf{5 0 S t 7 5 0}}$ is synthesized by polymerization of $0.3 \mathrm{~g}(50 \mathrm{wt} \%)$ St-PEG-750 and $0.3 \mathrm{~g}(50 \mathrm{wt} \%)$ of DVB.

Molecular weight of St-PEG-750 = 895

Weight fraction of PEG in St-PEG-350 $=(750 / 895)=0.838$

$0.3 \mathrm{~g}$ of St-PEG-750 contains $(0.3 * 0.838)=0.251 \mathrm{~g}$ of PEG

Assuming full extraction, the volume of PEG present in the sample should be equal to the pore volume.

Volume of PEG present in the sample $=(0.251 / 1.125)=\mathbf{0 . 2 2 3} \mathbf{~ c c}$

After the extraction, the remaining weight of the sample $=\{0.30+(0.3-0.251)\}=0.349 \mathrm{~g}$

Theoretical pore volume of $50 \mathrm{St} 750$ after extraction $=(0.223 / 0.349)=\underline{\mathbf{0 . 6 3}} \mathbf{~ c c} / \mathbf{g}$.

$\underline{\mathbf{5 0 S t 2 0 0 0}}$ is synthesized by polymerization of $0.3 \mathrm{~g}(50 \mathrm{wt} \%)$ St-PEG-2000 and $0.3 \mathrm{~g}$ (50 wt $\%$ ) of DVB.

Molecular weight of St-PEG-750 = 2145

Weight fraction of PEG in St-PEG-350 $=(2000 / 2145)=0.932$

$0.3 \mathrm{~g}$ of St-PEG-750 contains $(0.3 * 0.932)=0.279 \mathrm{~g}$ of PEG

Assuming full extraction, the volume of PEG present in the sample should be equal to the pore volume.

Volume of PEG present in the sample $=(0.279 / 1.125)=\mathbf{0 . 2 4 8} \mathbf{c c}$

After the extraction, the remaining weight of the sample $=\{0.30+(0.3-0.279)\}=0.321 \mathrm{~g}$

Theoretical pore volume of 50St2000 after extraction $=(0.248 / 0.321)=\underline{\mathbf{0 . 7 7}} \mathbf{c c} / \mathbf{g}$.

$\underline{\text { 50DiSt600 }}$ is synthesized by polymerization of $0.3 \mathrm{~g}$ (50 wt\%) Di-St-PEG-600 and 0.3g (50 wt $\%)$ of DVB.

Molecular weight of Di-St-PEG-600 = 890

Weight fraction of PEG in Di-St-PEG-600 $=(600 / 890)=0.6741$

$0.3 \mathrm{~g}$ of Di-St-PEG-600 contains $(0.3 * 0.6741)=0.202 \mathrm{~g}$ of PEG

Assuming full extraction, the volume of PEG present in the sample should be equal to the pore volume. 
Volume of PEG present in the sample $=(0.202 / 1.125)=\mathbf{0 . 1 8} \mathbf{~ c c}$

After the extraction, the remaining weight of the sample $=\{0.30+(0.3-0.202)\}=0.398 \mathrm{~g}$

Theoretical pore volume of 50DiSt600 after extraction $=(0.18 / 0.398)=\underline{\mathbf{0 . 4 5 2}} \mathbf{c c} / \mathbf{g}$.

$\underline{\text { 50DiSt1500 }}$ is synthesized by polymerization of $0.3 \mathrm{~g}(50 \mathrm{wt} \%)$ Di-St-PEG-1500 and $0.3 \mathrm{~g}$ (50 wt $\%)$ of DVB.

Molecular weight of Di-St-PEG-1500 = 1790

Weight fraction of PEG in Di-St-PEG-1500 $=(1500 / 1790)=0.8379$

$0.3 \mathrm{~g}$ of Di-St-PEG-1500 contains $(0.3 * 0.8379)=0.251 \mathrm{~g}$ of PEG

Assuming full extraction, the volume of PEG present in the sample should be equal to the pore volume.

Volume of PEG present in the sample $=(0.251 / 1.125)=\mathbf{0 . 2 2 3} \mathbf{~ c c}$

After the extraction, the remaining weight of the sample $=\{0.30+(0.3-0.251)\}=0.349 \mathrm{~g}$

Theoretical pore volume of 50DiSt1500 after extraction $=(0.223 / 0.349)=\underline{\mathbf{0 . 6 3 9}} \mathbf{c c} / \mathbf{g}$.

50PEG350 is synthesized by polymerization of $0.3 \mathrm{~g}(50 \mathrm{wt} \%) \mathrm{mPEG} 350$ and $0.3 \mathrm{~g}(50 \mathrm{wt} \%)$ of DVB.

Molecular weight of mPEG350 $=350$

Assuming full extraction, the volume of PEG present in the sample should be equal to the pore volume.

Volume of PEG present in the sample $=(0.3 / 1.125)=\mathbf{0 . 2 6 6} \mathbf{c c}$

After extraction the remaining weight of the sample $=0.3 \mathrm{~g}$

Theoretical pore volume of 50PEG350 $=(0.266 / 0.3)=\underline{\mathbf{0 . 8 8}} \mathbf{~ c c / g}$. 